\title{
Clinical phenotypes of SARS-CoV-2: implications for clinicians and researchers
}

\author{
Jordi Rello ${ }^{1,2,3}$, Enrico Storti ${ }^{4}$, Mirko Belliato ${ }^{5}$ and Ricardo Serrano ${ }^{6}$ \\ Affiliations: ${ }^{1}$ Centro de Investigación Biomedica en Red (CIBERES), Instituto de Salud Carlos III, Madrid, \\ Spain. ${ }^{2}$ CRIPS, Vall d'Hebron Institute of Research, Barcelona, Spain. ${ }^{3}$ Clinical Research, CHU Nîmes, \\ Université Montpellier-Nîmes, Nîmes, France. ${ }^{4}$ Anesthesia and ICU Dept, Hospitale de Lodi, Lodi, Italy. ${ }^{5}$ UOC \\ Anestesia e Rianimazione 1, Fondazione IRCCS Policlinico San Matteo, Pavia, Italy. ${ }^{6}$ Critical Care Dept, \\ Hospital de Hellin, Albacete, Spain.
}

Correspondence: Jordi Rello, Ps Vall d' Hebron 129, AMI-14, 08035 Barcelona, Spain. E-mail: jrellodcrips.es

@ERSpublications

SARS-CoV-2 infections present different specific individual phenotypes. Applying a personalised approach would benefit in optimisation of therapies and outcome improvement. \#COVID19 https:// bit.ly/3akTSuf

Cite this article as: Rello J, Storti E, Belliato M, et al. Clinical phenotypes of SARS-CoV-2: implications for clinicians and researchers. Eur Respir J 2020; 55: 2001028 [https://doi.org/10.1183/13993003.01028-2020].

ABSTRACT Patients with COVID-19 present a broad spectrum of clinical presentation. Whereas hypoxaemia is the marker of severity, different strategies of management should be customised to five specific individual phenotypes. Many intubated patients present with phenotype 4, characterised by pulmonary hypoxic vasoconstriction, being associated with severe hypoxaemia with "normal" $\left(>40 \mathrm{~mL} \cdot \mathrm{cmH}_{2} \mathrm{O}^{-1}\right.$ ) lung compliance and likely representing pulmonary microvascular thrombosis. Phenotype 5 is often associated with high plasma procalcitonin and has low pulmonary compliance, Which is a result of co-infection or acute lung injury after noninvasive ventilation. Identifying these clinical phenotypes and applying a personalised approach would benefit the optimisation of therapies and improve outcomes. 
The clinical spectrum of severe acute respiratory syndrome coronavirus 2 (SARS-CoV-2) infection is broad, ranging from asymptomatic infection to flu-like illness (sometimes with digestive disturbances) to viral pneumonia. Patients with pneumonia may have only minor opacification with near normal chest radiographs but have the potential to develop an acute respiratory failure with severe hypoxaemia of quick progression. The need for mechanical ventilation can be identified by increases in respiratory rates and requirement of high oxygen concentrations to maintain arterial oxygen saturation $>90 \%$. However, due to the scenario of the crisis with limited resources, many patients have experienced intubation delay after failure of continuous positive airway pressure, noninvasive ventilation or high-flow nasal oxygen therapy. Thus, the estimation of risk factors for mechanical ventilation have not been well identified due to the lack of standardisation of practices. This article reports clinical experience from the SARS-CoV-2 surge acquired from different hospitals in Italy and Spain (on 27 March 2020) and is supported by clinical observations. Further validation with stronger evidences is required.

As with influenza, reverse transcriptase-PCR for SARS-CoV-2 is associated with frequent false-negative results [1]. Therefore, patients may be diagnosed by a characteristic computed tomography (CT) scan. It is important to advise that high procalcitonin should not exclude the diagnosis, because it has been often reported in children and young adults and often suggests co-infection with a bacterial pathogen [2]. Ageing, hypertension and diabetes have been consistently reported as risk factors for death or severity, whereas pregnancy does not seem to be a risk factor like in influenza [3]. Current reported information may have different profiles beyond China, affecting younger or obese subjects due to differences in prevalence and socialisation. In China, SARS-CoV-2 has not shown a more severe disease in immunocompromised patients. That is in contrast with other respiratory viral agents, such as influenza, respiratory syncytial virus, adenovirus or rhinovirus; possibly because in coronavirus the host innate immune response appears to be the main driver of lung tissue injury during viral infection [4]. Caution should still be advised with immunocompromised patients as the total number of reported cases in this subgroup remains low.

Clinical and laboratory features are characteristic with lymphocytopenia, elevation of lactate dehydrogenase, high plasma C-reactive protein and low procalcitonin [5]. Many patients have hypercoagulability with complement mediated micro-angiopathy, increasing the risk of deep venous thrombosis, pulmonary artery micro-thrombosis, distal arterial micro-thrombosis affecting fingers, and cardiovascular events such as myocardial ischaemia, ischaemic stroke and pulmonary embolisms. In severe cases there can be dramatic elevation of D-dimer values, reduced fibrinogen with a high international normalised ratio and low platelet count, associated with the induction of tissue factor expression. Some intensive care unit patients in China reported by ZHANG et al. [6] showed antiphospholipid autoimmune responses that led to thrombotic events, but lupus anticoagulant was not detected. Endothelial damage leading to inflammation and thrombosis plays a big role. Thus, all coronavirus 2019 (COVID-19) hospitalised patients are tributary of benefit from prophylactic anticoagulation, unless they have severe thrombocytopenia or active bleeding. Long chain (unfractionated) heparin would theoretically be preferable because of their anti-inflammatory effects. Prescribing heparin to those patients with 6-fold levels of D-dimers above upper normal ranges has been associated with significantly reduced 28 -day mortality rates ( $52 \%$ versus $32 \%, \mathrm{p}=0.01$ ) among 449 patients with severe SARS-CoV-2 infection in China [7]. This scenario may suggest an alteration of the microvascular endothelium or a secondary complement mediated micro-angiopathy activated by the virus or by an associated cytokine storm. ZHOU et al. [5] reported 54 nonsurvivors with median serum ferritin $>1400 \mathrm{ng} \cdot \mathrm{L}^{-1}$. A few of these patients require bone marrow aspirate and may develop hemophagocytic lymphohistiocytosis syndrome, requiring $\gamma$-globulins and high-dose dexamethasone. As the current experience with endemic human coronavirus pneumonia is that it may double 12-month mortality [8], patients should be monitored after clinical cure to assess for persistent hyperinflammation or hypercoagulability and consideration for preventive interventions.

In an era of precision medicine, it is important to identify the main phenotypes in order to customise therapy in a personalised approach [9]. A variety of proposed anti-viral, anti-inflammatory, anticoagulant and anti-fibrotic strategies may be beneficial or harmful depending on the state of disease. Recognition of different phenotypes of SARS-CoV-2 is therefore critical to clinicians and researchers for timely, effective and safe therapeutic interventions.

The most benign phenotype (phenotype 1) is the most common and only symptomatic therapy needs to be considered, which is characterised by fever, headache or mild respiratory symptoms (like cough or sore throat) and malaise, but the chest radiograph is normal and hypoxaemia is not present. Phenotype 2 represents $80 \%$ of hospitalisations and is characterised by the presence of hypoxaemia or small opacities on chest radiographs and these patients should be referred for close respiratory monitoring (particularly respiratory rate and oxygen saturation measure by pulse oximetry) because they are at risk of rapid 
deterioration progressing to death if intubation is not timely instituted. These patients are typically hyper-inflamed and remain hypovolemic at presentation to the hospital. Thus, restriction of furosemide use should be considered, except if they have received a huge volume infusion.

Phenotype 3 represented 15\% of hospitalisations in China [10] and presents with greater hypoxaemia and higher respiratory rates ( 30 breaths $\cdot \mathrm{min}^{-1}$ or 25 breaths $\cdot \mathrm{min}^{-1}$ in those aged $>55$ years of age or healthy subjects $<55$ years of age, respectively). Patients may present as phenotype 3 or progress from phenotype 2 because hypoxaemia progresses quickly. High interleukin-6 is a biomarker which may help to differentiate these two phenotypes. Phenotypes 2 and 3 are good candidates for randomised clinical trials to assess the efficacy of drugs with either anti-viral activity (to block replication very early), anti-inflammatory drugs or anti-fibrotic drugs. In our opinion, delaying intubation using noninvasive ventilation may induce acute lung injury. Given the good lung compliance and low work of breathing, these patients may avoid mechanical ventilation despite requirement for high inspiratory oxygen fraction, using awake prone position whereas they have spontaneous breathing. Arterial oxygen tension/inspiratory oxygen fraction ratios should not be a criteria for intubation. Intubation should be clinically considered in the presence of respiratory alkalosis with progressive hyperventilation when delivering high oxygen concentrations.

Phenotype 4 is characterised by severe hypoxaemia requiring intubation, under strict measures to limit aerosolisation. CT scans document oedema in the lower lobes. Angio-CT in patients with multiple ground-glass opacities often show micro-embolic lesions. Lung ultrasonography is consistent with interstitial injury with B lines (white lung) [11]. In the presence of reduced sliding and multiple subpleural opacities, intubation should not be delayed, particularly when $\mathrm{Bb}$ coalescent lines are present in all evaluated zones or in the majority (more than three fields explored). This phenotype is characterised by an organising pneumonia with hypoxic vasoconstriction associated with severe hypoxaemia. Nitric oxide or prostacyclin, when available, should be considered as potential rescue therapies. This phenotype has "normal" $\left(>40 \mathrm{~mL} \cdot \mathrm{cmH}_{2} \mathrm{O}^{-1}\right.$ ) lung compliance and likely does not represent acute respiratory distress syndrome. The target should be to maintain a venous oxygen saturation $>90 \%$, avoiding ventilatory asynchronies. High positive end-expiratory pressure (PEEP) in patients with normal compliance may have detrimental effects on haemodynamics [12]. PEEP should be enough to be maintained at $8-10 \mathrm{cmH}_{2} \mathrm{O}$, with higher levels compromising cardiovascular stability. Most patients had prior hyperinflammation and hyperpyrexia, and are hypovolemic. Under these conditions, PEEP values $>10 \mathrm{cmH}_{2} \mathrm{O}$ may be harmful, often being associated with a need for high dose vasopressors and acute kidney injury, thus complicating management. Respiratory rate should be $<20$ beats. $\min ^{-1}$. Recruitment manoeuvres are not beneficial and are contraindicated. Prone positioning/supine cycles are of little benefit in this phenotype, contributing to increased risks of contamination and fatigue of healthcare workers. In this phenotype, tidal volumes $>6 \mathrm{~mL} \cdot \mathrm{kg}^{-1}$ are likely to be less harmful than in typical acute respiratory distress syndrome. GATTiNONi et al. [12] suggest that tidal volumes of $6-9 \mathrm{~mL} \cdot \mathrm{kg}^{-1}$ may be allowed in order to control hypercapnia because the risk of

\section{TABLE 1 Clinical phenotypes of SARS-CoV-2 disease}

Type Proportion Clinical features

$180-85 \%$ of symptomatic patients

2

3 $\sim 15 \%$ of hospitalised patients

4 $\sim 2 / 3$ of patients needing mechanical ventilation

5 $\sim 1 / 3$ of patients needing mechanical ventilation
Fever, headache, mild respiratory symptoms, sore throat, no hypoxaemia, normal chest radiograph, excellent prognosis

Mild hypoxia, minor (usually bilateral) infiltrates on chest radiograph, up to $15 \%$ may progress quickly to type 3

Moderate to severe hypoxaemia and tachypnoea, high IL-6 and other inflammatory markers

May progress to types 4 or 5

Severe hypoxaemia requiring mechanical ventilation, normal lung compliance, good response to nitric oxide, prone position of little benefit, tidal volume $>6 \mathrm{~mL} \cdot \mathrm{kg}^{-1}$ allowed, respiratory rate $<20$ beats $\cdot \min ^{-1}$, PEEP $<10 \mathrm{~cm} \mathrm{H}_{2} \mathrm{O}$

High procalcitonin may be increased if mechanical ventilation is delayed in severely hypoxaemic patients, more in keeping with classical ARDS

Protective ventilatory strategy and prone position indicated

SARS-CoV-2: severe acute respiratory syndrome coronavirus 2; IL-6: interleukin-6; PEEP: positive end-expiratory pressure; ARDS: acute respiratory distress syndrome. 
TABLE 2 Clinical data from the first patients requiring mechanical ventilation at the Hospital Hellin (Albacete, Spain) intensive care department

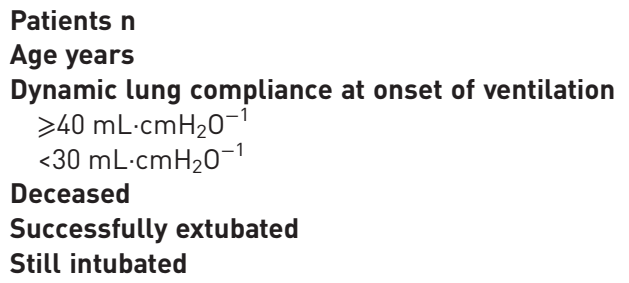

Data are presented as n, mean (range) or $\mathrm{n}(\%)$. Patients were assessed on 5 April 2020.

ventilator-induced lung injury may be better tolerated if high lung compliances are maintained. These observations are consistent with a preliminary analyses of 16 patients [13].

Phenotype 5 is less common than phenotype 4 (table 1) and it is an advanced stage with associated acute lung injury. Co-infection or acute lung injury is associated with increased plasma procalcitonin levels, which may help to distinguish it. In our experience, this phenotype was rarely documented in patients who underwent rapid intubation (table 2). Typically, it was present in patients with SARS-CoV-2 infection who were started on noninvasive ventilation (via helmet in Italy or facemask in China) but less common in patients started on ventilation promptly [14]. Oxygenation should be assessed measuring the shunt fraction. Lung compliance showed values $<40 \mathrm{~mL} \cdot \mathrm{cmH}_{2} \mathrm{O}^{-1}$. This phenotype may benefit from high PEEP values and prone positioning, with a similar management strategy to acute respiratory distress syndrome. The potential benefit of immunomodulatory agents at this stage may result in the emergence of severe adverse events which complicate management, such as systemic super infections (viremia by cytomegalovirus and bacterial sepsis).

In summary, patients with COVID-19 present a broad spectrum of clinical presentations. Whereas hypoxaemia is the marker of severity, different strategies of management should be customised to the specific individual phenotypes (table 1). Applying a personalised approach would benefit the optimisation of therapies and improve outcomes.

Conflict of interest: J. Rello has nothing to disclose. E. Storti has nothing to disclose. M. Belliato reports personal fees for lectures from Hamilton Medical, Swiss and Bonaduz, outside the submitted work. R. Serrano has nothing to disclose.

\section{References}

1 Walter JM, Wunderink RG. Testing for respiratory viruses in adults with severe lower respiratory infections. Chest 2018; 154: 1213-1222.

2 Xia W, Shao J, Guo Y, et al. Clinical and CT features in pediatric patients with COVID-19 infection: different points from adults. Pediatr Pulmonol 2020; 55: 1169-1174.

3 Maraví-Poma E, Martin-Loeches I, Regidor E, et al. Severe 2009 A/H1N1v influenza in pregnant women in Spain. Crit Care Med 2011; 39: 945-951.

4 D'Antiga L. Coronaviruses and immunosuppressed patients. The facts during the third epidemic. Liver Transpl 2020; in press [https://doi.org/10.1002/lt.25756].

5 Zhou F, Yu T, Du R, et al. Clinical course and risk factors for mortality of adult inpatients with COVID-19 in Wuhan, China: a retrospective cohort study. Lancet 2020; 395: 1054-1062.

6 Zang Y, Xiao M, Zhang S, et al. Coagulopathy and antiphospholipid antibodies in patients with covid-19. N Engl J Med 2020; 382: e38.

7 Tang N, Bai $\mathrm{H}$, Chen $\mathrm{X}$, et al. Anticoagulant treatment is associated with decreased mortality in severe coronavirus disease in patients with coagulopathy. J Thromb Haemost 2020; 18: 1094-1099.

8 Arnold F, Burns M, Mahmood $\mathrm{K}$, et al. Endemic human coronaviruses in hospitalized adults with community-acquired pneumonia: results from the Louisville Pneumonia Study. J Respir Infect 2020; 4: 1.

9 Rello J, van Engelen TSR, Alp E, et al. Towards precision medicine in sepsis: a position paper from the European Society of Clinical Microbiology and Infectious Diseases. Clin Microbiol Infect 2018; 24: 1264-1272.

10 Rello J, Tejada S, Userovici C, et al. Coronavirus disease 2019 (COVID-19): a critical care perspective beyond China. Anaesth Crit Care Pain Med 2020; 39: 167-169.

11 Sanjan A, Krishnan SV, Abraham SV, et al. Utility of point-of-care lung ultrasound for initial assessment of acute respiratory distress syndrome patients in the emergency department. J Emerg Trauma Shock 2019; 12: 248-253.

12 Gattinoni L, Chiumello D, Caironi P, et al. COVID-19 pneumonia different respiratory treatment for different phenotypes? Intensive Care Med 2020; in press [https://doi.org/10.1007/s00134-020-06033-2].

13 Gattinoni L, Coppola S, Cressoni M, et al. COVID-19 does not lead to a "typical" acute respiratory distress syndrome. Am J Respir Crit Care Med 2020; in press [https://doi.org/10.1164/rccm.202003-0817LE].

14 Barrasa H, Rello J, Tejada S, et al. SARS-CoV-2 in Spanish intensive care: early experience with 15-day survival in Vitoria. Anesth Crit Care Pain Med 2020; in press [https://doi.org/10.1016/j.accpm.2020-04.001]. 